\title{
Hipovitaminose A em escolares da zona rural de Minas Gerais ${ }^{1}$
}

\author{
Vitamin A deficiency in school children of the \\ rural area in Minas Gerais, Brazil
}

Margarete Aparecida SANTOS²

Eliane Garcia REZENDE 3

Joel Alves LAMOUNIER ${ }^{4}$

Márcio Antônio Moreira GALVÃO2

Élido BONOMO²

Romário Cerqueira LEITE ${ }^{5}$

\section{R E S U M O}

\section{Objetivo}

Com o presente estudo, procurou-se identificar a prevalência da hipovitaminose A em escolares da zona rural do município de Novo Cruzeiro, MG, bem como possíveis fatores predisponentes para sua ocorrência.

\section{Métodos}

A amostra foi constituída de 241 crianças, de seis a catorze anos de idade, de quatro escolas rurais. Os níveis séricos de retinol foram interpretados pelos critérios do Interdepartmental Committee on Nutrition for National Defense. A importância epidemiológica da hipovitaminose A foi avaliada segundo os critérios da Organização Mundial da Saúde. Foram adotados como fatores predisponentes da hipovitaminose A, as parasitoses intestinais, a desnutrição energético-protéica, o consumo inadequado de fontes de vitamina $\mathrm{A}$ e a renda familiar per capita. Por se tratar de um estudo transversal, com variáveis dicotômicas, empregou-se o teste qui-quadrado na análise estatística.

\section{Resultados}

Identificou-se a hipovitaminose A em 29,0\% dos indivíduos estudados; 23,2\% deles apresentaram desnutrição pregressa (stunting), 8,7\% eram desnutridos segundo o índice de massa corporal; em 63,1\% dos casos identificou-se inadequação no consumo de fontes alimentares de vitamina A e 78,8\% dos escolares eram portadores de algum tipo de parasita intestinal. A maioria das famílias dos escolares $(87,1 \%)$ tinha renda

\footnotetext{
1 Artigo baseado na tese "Fatores predisponentes para hipovitaminose A em escolares da zona rural do município de Novo Cruzeiro, Minas Gerais". Escola de Medicina Veterinária, Universidade Federal de Minas Gerais, 2002. 88p.

2 Escola de Nutrição, Departamento de Nutrição Clínica e Social, Universidade Federal de Ouro Preto. Campus Universitário s/n, Morro do Cruzeiro, 35400-000, Ouro Preto, MG, Brasil.

3 Escola de Farmácia e Odontologia de Alfenas, Centro Universitário Federal. Alfenas, MG, Brasil.

${ }^{4}$ Faculdade de Medicina, Universidade Federal de Minas Gerais. Belo Horizonte, MG, Brasil.

5 Escola de Veterinária, Universidade Federal de Minas Gerais. Belo Horizonte, MG, Brasil.
} 
mensal per capita de até um quarto do salário mínimo; as demais famílias apresentavam-se, 10,4\%, na faixa de renda per capita entre um quarto de salário e meio salário mínimo inclusive; 2,1\%, na faixa entre meio salário e um salário mínimo inclusive; e apenas 0,4\% das famílias, na faixa de renda maior que um salário mínimo.

\section{Conclusão}

Concluiu-se que a hipovitaminose A é um problema de saúde pública entre os escolares. Não se observou associação estatisticamente significante entre hipovitaminose A e os fatores predisponentes estudados.

Termos de Indexação: causalidade, hipovitaminose A, escolares.

\section{A B S T R A C T}

\section{Objective}

The objective of this study was to verify the occurrence of vitamin A deficiency in school children of the rural area of Novo Cruzeiro, Minas Gerais, Brazil, as well as to identify the possible predisposing factors for such occurrence.

\section{Methods}

The sample comprised 241 school children, ranging from 6 to 14 years of age, from four rural schools of the region. The serum levels of retinol were interpreted by the criteria of the Interdepartmental Committee on Nutrition National Defense. The epidemiological significance of the vitamin A deficiency was evaluated according to the World Health Organization criteria. As predisposing factors for vitamin A deficiency, the following conditions were considered: intestinal parasitism, protein-energy malnutrition, inadequate ingestion of vitamin A food sources, and per capita family income. Statistical analysis was carried out using Chi-square test.

\section{Results}

Vitamin A deficiency was identified in $29.0 \%$ of the subjects, $23.2 \%$ of the children presented stunting, and $8.7 \%$ were malnourished, according to the body mass index. In $63.1 \%$ of the subjects, inadequate ingestion of retinol sources was verified, while $78.8 \%$ of the subjects presented some type of intestinal parasite. Most school-children families (87.1\%) had per capita monthly incomes bellow $1 / 4$ of the minimum wage; the rest of the families were situated respectively in the ranges: $(10.4 \%)>1 / 4$ to $\leq 1 / 2$ minimum wage; $(2.1 \%)>1 / 2$ to $\leq 1$ minimum wage; and ( $0.4 \%)>1$ minimum wage.

\section{Conclusion}

Vitamin A deficiency among school children was found to be a public health problem in the studied area. Nonetheless, no significant statistic association between vitamin A deficiency and the factors selected as predisposing ones was observed.

Indexing terms: causality, Vitamin A deficiency, school children.

\section{N T R O D U Ç Ã O}

Estudos realizados no Brasil demonstraram que a deficiência de vitamina A é um importante problema de saúde ${ }^{1,2}$. Esses estudos têm identificado prevalências superiores a $10 \%$ de níveis de retinol sérico abaixo de $20 \mu \mathrm{g} / \mathrm{dL}$, condição que caracteriza a hipovitaminose A como problema de saúde pública³.

A importância da identificação de hipovitaminose $A$ na infância reside no fato de a vitamina A exercer importantes e numerosas funções no organismo e, portanto, a sua defi- ciência acarretar conseqüências fisiopatológicas para o indivíduo, principalmente em crianças. Dentre as repercussões, encontra-se aquela relacionada com a "secura" da conjuntiva e o decréscimo da visão noturna provocadas por deficiência moderada de vitamina A, podendo evoluir para a cegueira irreversível ${ }^{4}$. As evidências indicam também que essa deficiência pode comprometer o crescimento de crianças ${ }^{5}$ e diminuir a resistência às infecções ${ }^{4,6}$.

De acordo com dados compilados pela Organização Mundial de Saúde (OMS), no Brasil 
são mais freqüentes as formas subclínicas da deficiência de vitamina A e, embora os pré-escolares constituam o grupo mais vulnerável para a hipovitaminose $A$, sua ocorrência pode se prolongar para a idade escolar e a fase adulta ${ }^{7}$. No entanto, no Brasil, a maioria dos estudos é conduzida entre pré-escolares e são escassos aqueles que envolvem os escolares, principalmente os da zona rural, que constituem uma importante parcela da população brasileira em risco de desenvolver essa deficiência nutricional.

O estado de saúde e nutrição é reflexo das condições materiais de vida e do padrão de morbidade a que está submetida a população. MacLaren \& Frigg ${ }^{8}$, através de extensa revisão de literatura, apontam a idade - principalmente a dos pré-escolares; o estado fisiológico - principalmente aquele que demanda maior disponibilidade de vitamina A, como a gravidez; a inadequação da ingestão alimentar; a ocorrência de desmame precoce; as doenças infecciosas; a desnutrição; a baixa condição socioeconômica e o sexo masculino, como fatores que, de alguma forma, estão implicados na etiopatogenia da hipovitaminose $\mathrm{A}$.

A cidade de Novo Cruzeiro, localizada no Vale do Jequitinhonha, região reconhecida como de alta prevalência de desnutrição e doenças infecciosas em decorrência da pobreza a que está submetida sua população, é detentora de uma precária condição de vida, conforme ressaltado pelo índice de desenvolvimento humano (IDH) - calculado pela média simples do índice de longevidade, educação e renda - de 0,629, segundo o Instituto de Pesquisa Econômica Aplicada (IPEA), que o coloca no $791^{\circ}$ lugar entre os 853 municípios mineiros. O mapa da fome ${ }{ }^{9}$, elaborado pelo IPEA em 1993, revela a existência, nesse município, de 2900 famílias que vivem abaixo do nível de pobreza, conforme critérios desse instituto ${ }^{10}$.

Reconhecendo que a hipovitaminose A é problema importante de saúde entre as crianças que estão submetidas às precárias condições de vida, e pretendendo fornecer subsídios para programas de vigilância epidemiológica, realizou-se este estudo visando verificar a ocorrência e os fatores predisponentes da hipovitaminose A em escolares da zona rural do município de Novo Cruzeiro, localizado no Vale do Jequitinhonha, em Minas Gerais.

\section{M É T O D OS}

Este é um estudo de natureza transversal e integra uma pesquisa mais ampla desenvolvida em Novo Cruzeiro, MG, em 1999, pela Universidade Federal de Ouro Preto (UFOP) e pela Universidade Federal de Minas Gerais (UFMG), contando com o apoio de técnicos da Fundação Ezequiel Dias (FUNED), da Fundação Nacional de Saúde, da Diretoria Regional de Saúde (DRS) de Teófilo Otoni e da Secretaria de Saúde de Novo Cruzeiro, MG.

O município de Novo Cruzeiro, MG, tem uma população de 30453 habitantes distribuída numa área de $1701 \mathrm{~km}^{2}$, implicando em densidade demográfica de 17,9hab/ $\mathrm{km}^{2}$. A maior parte da população - aproximadamente 22075 habitantes - reside na região rural ${ }^{11}$.

Foram elegíveis para o estudo 430 escolares de seis a catorze anos de idade matriculados em quatro escolas da zona rural. No entanto, muitos dos escolares não compareceram no momento da coleta de dados, além de ocorrência de perdas sangüíneas por hemólise, implicando em viéses de não resposta. Dessa forma, a amostra foi constituída por 241 escolares, cujas variáveis de interesse estavam disponíveis.

Para estudar o fenômeno, foi selecionado como variável dependente o retinol sérico e, como variáveis independentes, o estado antropométrico, a ingestão de alimentos fontes de vitamina $A$, a renda familiar mensal per capita e parasitoses intestinais.

A pesquisa foi aprovada pelo Comitê de Ética e Pesquisa (CEP) da UFOP, em atendimento à Resolução n 196/1996 do CNS do Ministério da Saúde. 
Conforme exigências éticas da pesquisa, após o conhecimento dos objetivos do estudo, os pais ou responsáveis pela criança assinaram o termo de consentimento, condição necessária para a participação do escolar no estudo.

A coleta e o manuseio de amostras de sangue foram realizados por profissionais especializados da Secretaria Estadual de Saúde, da Diretoria Regional de Saúde de Teófilo Otoni e da Fundação Ezequiel Dias. As amostras de sangue foram coletadas por venopuntura em vacuntainers, em ambiente semi-escurecido, transportadas ao laboratório montado em campo e centrifugadas imediatamente. Uma vez separado, o soro foi protegido da exposição à luz, ao ar, sendo congelado a uma temperatura de $-80^{\circ} \mathrm{C}$ até o momento da dosagem. O retinol sérico foi determinado pelo método espectrofotométrico ${ }^{12}$ no Laboratório da Escola de Nutrição da UFOP.

Os níveis séricos de retinol foram classificados, segundo critérios do Interdepartmental Committee on Nutrition for National Defense, em: alto $(>50,0 \mu \mathrm{g} / \mathrm{dL})$, aceitável $(20,0$ a $49,9 \mu \mathrm{g} / \mathrm{dL})$, baixo $(19,9$ a $10,0 \mu \mathrm{g} / \mathrm{dL})$ e deficiente $(<10,0 \mu \mathrm{g} / \mathrm{dL})^{13}$. Foram considerados inadequados os níveis baixos e deficientes de retinol. A importância epidemiológica da hipovitaminose A foi definida segundo os limites estabelecidos pela World Health Organization ${ }^{3}$, os quais atribuem a classificação "leve" quando se observam percentuais de inadequação menores que 10\%; "moderado" quando os limites situam entre 10\% e $20 \%$ e "grave" quando os limites da inadequação ultrapassam $20 \%$.

Neste estudo, os fatores predisponentes da hipovitaminose A são representados pela desnutrição, consumo alimentar inadequado de fontes de vitamina A, baixa renda mensal familiar per capita e a parasitoses intestinais.

As medidas utilizadas para avaliação antropométrica foram o peso e a altura. Para medição do peso corporal, foi utilizada balança microeletrônica portátil, com capacidade de $150 \mathrm{~kg}$, da marca Filizola ${ }^{\circledR}$, utilizando-se escala de $0,1 \mathrm{~kg}$. Para tomada da altura, uma fita métrica fiber-glass foi afixada em parede lisa, plana, sem rodapé e em posição vertical, com escala de $0,1 \mathrm{~cm}$. A idade da criança foi calculada pela diferença entre a data de coleta de dados e a data de nascimento, obtida pelo registro escolar.

A avaliação antropométrica foi realizada por meio do software "EPI-NUTRI" (módulo de antropometria nutricional) do "Epi-Info", versão 6.04, baseado no National Center for Health Statistics/World Health Organization.

O indicador altura/idade foi adotado para avaliar o estado antropométrico pregresso do escolar. Consideram-se com déficit linear (stunting) crianças com $<-2$ desvios-padrão em escore-Z da média da referência do padrão do National Center for Health Statistics, conforme recomendado pela $\mathrm{OMS}^{7}$.

Para avaliar a adequação do peso em relação à altura, utilizou-se o índice de massa corporal (IMC). Indivíduos com valores de IMC abaixo do ponto de corte (percentil 5th) foram considerados como desnutridos ${ }^{14}$.

Empregou-se questionário de freqüência alimentar semiquantitativo ${ }^{15}$ adaptado para a pesquisa a fim de avaliar o consumo de vitamina A. Para garantir a veracidade das informações, foi solicitada a presença dos pais ou responsável pela criança no momento da entrevista.

As informações foram referentes à freqüência e à quantidade de ingestão de alimentos fontes de vitamina $A$. A freqüência do consumo foi categorizada em: uma vez por semana, duas a três vezes por semana, quatro a cinco vezes por semana, quinzenalmente, mensalmente ou raramente. Com base nas informações, obteve-se o escore de ingestão, ou seja, a proporção de uso diário correspondente. Multiplicando tal resultado pela quantidade da porção usual, obteve-se a média de consumo diário do nutriente investigado ${ }^{15}$.

O cálculo do consumo de vitamina A foi estimado com base na ingestão de carotenóides e de vitamina A pré-formada, convertidas em equivalentes de retinol. 
A quantidade média dos alimentos ingeridos (em gramas) foi lançada no programa Sistema de Análise Nutricional/Virtual Nutri, versão 1.0 para Windows ${ }^{\circledR}$ - 1996, do Departamento de Nutrição, Faculdade de Saúde Pública da Universidade de São Paulo, que permitiu a obtenção do consumo do nutriente. Adotou-se como valor adequado de ingestão de vitamina $A$ (equivalentes de retinol) aquele igual ou superior a 90\% das Recommended Dietary Allowances ${ }^{16}$.

As informações socioeconômicas foram coletadas empregando-se um questionário estruturado. A renda mensal familiar per capita foi obtida pela razão entre a renda total em salários mínimos e o número de residentes no domicílio, e foi categorizada em: renda $1: \geq 0$ a $\leq 1 / 4$; renda $2:>1 / 4$ a $\leq 1 / 2 ;$ renda $3:>1 / 2$ a $\leq 1$ e renda $4:>1$.

Foram entregues frascos para coleta de uma amostra de fezes por indivíduo, com as devidas orientações para o estudante ou pais/responsáveis sobre o manuseio do material. Realizou-se exame coproparasitológico pelo método Kato-Katz ${ }^{17}$ com o objetivo de identificar qualitativamente a presença de helmintos.

A prevalência, neste estudo, foi adotada como medida de ocorrência. Para testar a significância estatística, utilizou-se o teste do qui-quadrado. Adotou-se a significância de 0,05 para aceitar a associação entre os eventos estudados.

\section{RESULTA DOS}

Quanto à distribuição dos níveis séricos de retinol dos escolares investigados, identificou-se que $29 \%$ deles apresentaram níveis inadequados de retinol $(<20 \mu \mathrm{g} / \mathrm{dL})$ (Tabela 1). Observou-se que entre os escolares com níveis séricos de retinol adequados, 22,2\% apresentavam déficit de altura/ idade e 9,9\% apresentavam déficit ponderal. Verificou-se também que, dos indivíduos com níveis inadequados de retinol, 25,7\% apresentavam déficit de altura/idade e 5,7\% déficit ponderal. Não se observou associação significante entre tais variáveis e os níveis séricos de retinol (Tabela 2).

Entre os escolares investigados, 63,1\% apresentavam ingestão de vitamina A abaixo do recomendado (Tabela 3). Daqueles com níveis séricos adequados de retinol, 62,6\% apresentavam ingestão inadequada de vitamina $\mathrm{A}$ e $64,3 \%$ daqueles com níveis séricos inadequados de retinol apresentavam ingestão abaixo do recomendado. Não se observou associação significante entre níveis séricos de retinol e ingestão de vitamina $\mathrm{A}(p=0,918)$.

Quando a renda mensal familiar per capita foi investigada, verificou-se que $87,1 \%, 10,4 \%$, $2,1 \%$ e $0,4 \%$ dos indivíduos estavam situados, respectivamente, nas faixas entre zero inclusive e um quarto inclusive, um quarto e meio inclusive,

Tabela 1. Distribuição dos níveis séricos de retinol em escolares de Novo Cruzeiro, MG, 1999.

\begin{tabular}{lcc}
\hline Níveis séricos de retinol & $\mathrm{n}$ & $\%$ \\
\hline Adequados $(\geq 20 \mu \mathrm{g} / \mathrm{dL})$ & 171 & 71 \\
Inadequados $(<20 \mu \mathrm{g} / \mathrm{dL})$ & 70 & 29 \\
\hline Total & 241 & 100 \\
\hline
\end{tabular}

Tabela 2. Associação entre níveis séricos de retinol e estado antropométrico em escolares de Novo Cruzeiro, MG, 1999.

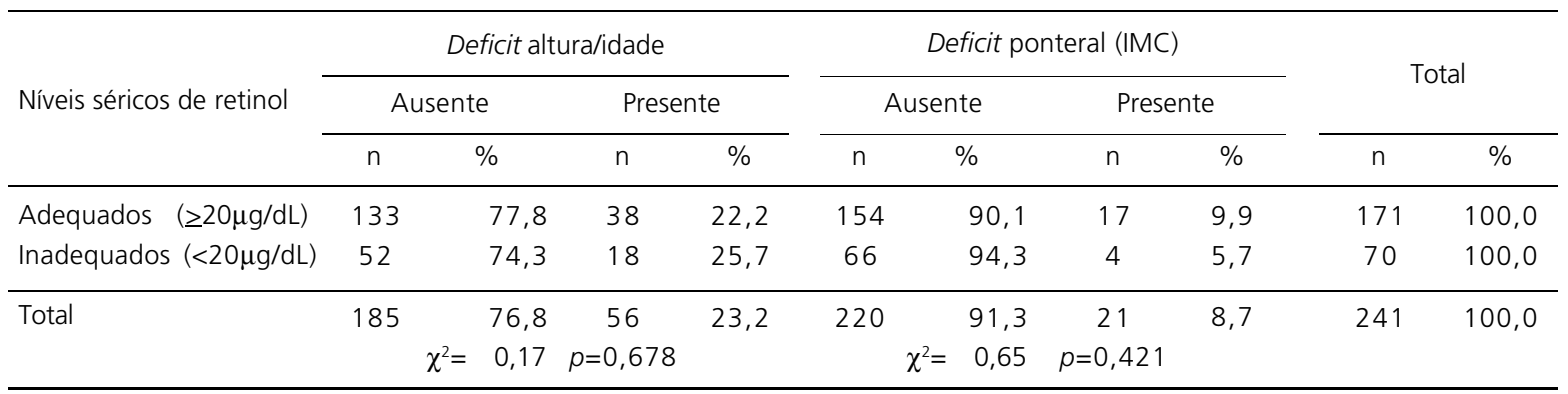


meio e um inclusive e maior que um salário mínimo. Ao associar renda com níveis séricos de retinol, notou-se que, dentre os indivíduos com níveis de retinol adequados, 85,4\% apresentavam renda familiar per capita menor ou igual a um quarto do salário mínimo, 11,1\% estavam entre os que possuíam renda entre um quarto e meio salário mínimo inclusive, 2,9\% entre meio e um inclusive e 0,6\% com renda familiar per capita acima de um salário mínimo. Daqueles com níveis de retinol sérico inadequados, $91,4 \%$ e $8,6 \%$ possuíam rendas familiares per capita situadas, respectivamente, nas faixas entre menor que um quarto inclusive e entre um quarto e meio salário mínimo inclusive. Aqueles com renda familiar per capita acima de meio salário mínimo não apresentaram hipovitaminose $A$. Não houve associação significante entre níveis séricos de retinol e renda familiar per capita entre os escolares investigados $(p=0,396)$ (Tabela 4).

Verificou-se (Tabela 5) que 78,8\% dos escolares apresentavam algum tipo de parasitose intestinal. Dentre os indivíduos portadores de níveis séricos de retinol adequados, $76,6 \%$ eram

Tabela 3. Associação entre níveis séricos de retinol e ingestão de vitamina A em escolares de Novo Cruzeiro, MG, 1999.

\begin{tabular}{|c|c|c|c|c|c|c|}
\hline \multirow{3}{*}{ Níveis séricos de retinol } & \multicolumn{4}{|c|}{ Ingestão de vitamina A } & \multirow{2}{*}{\multicolumn{2}{|c|}{ Total }} \\
\hline & \multicolumn{2}{|c|}{ Adequada } & \multicolumn{2}{|c|}{ Inadequada } & & \\
\hline & $n$ & $\%$ & $n$ & $\%$ & $\mathrm{n}$ & $\%$ \\
\hline Adequados $(\geq 20 \mu \mathrm{g} / \mathrm{dL})$ & 64 & 37,4 & 107 & 62,6 & 171 & 100,0 \\
\hline Inadequados $(<20 \mu \mathrm{g} / \mathrm{dL})$ & 25 & 35,7 & 45 & 64,3 & 70 & 100,0 \\
\hline \multirow[t]{2}{*}{ Total } & 89 & 36,9 & 152 & 63,1 & 241 & 100,0 \\
\hline & & 0,01 & $p=0,918$ & & & \\
\hline
\end{tabular}

Tabela 4. Associação entre níveis séricos familiar per capita, em escolares de Novo Cruzeiro, MG, 1999.

\begin{tabular}{|c|c|c|c|c|c|c|}
\hline \multirow{3}{*}{ Renda familiar per capita } & \multicolumn{4}{|c|}{ Níveis séricos de retinol } & \multirow{2}{*}{\multicolumn{2}{|c|}{ Total }} \\
\hline & \multicolumn{2}{|c|}{ Adequada } & \multicolumn{2}{|c|}{ Inadequada } & & \\
\hline & $\mathrm{n}$ & $\%$ & $\mathrm{n}$ & $\%$ & $\mathrm{n}$ & $\%$ \\
\hline$\geq 0 \leq 1 / 4$ salário mínimo & 146 & 85,4 & 64 & 91,4 & 210 & 87,1 \\
\hline$>1 / 4 \leq \leq 1 / 2$ salário mínimo & 19 & 11,1 & 6 & 8,6 & 25 & 10,4 \\
\hline$>\frac{1}{2} \leq 1$ salário mínimo & 5 & 2,9 & 0 & 0 & 5 & 2,1 \\
\hline > 1 salário mínimo & 1 & 0,6 & 0 & 0 & 1 & 0,4 \\
\hline \multirow[t]{2}{*}{ Total } & 171 & 100,0 & 70 & 100,0 & 241 & 100,0 \\
\hline & & $\chi^{2}=2,97$ & & $\mathrm{GL}=3$ & & $p=0,396$ \\
\hline
\end{tabular}

Obs: salário mínimo em $1999=\mathrm{R} \$ 180,00$.

Tabela 5. Associação entre níveis séricos de retinol e parasitoses intestinais, em escolares de Novo Cruzeiro, MG, 1999.

\begin{tabular}{|c|c|c|c|c|c|c|}
\hline \multirow{2}{*}{ Níveis séricos de retinol } & \multicolumn{4}{|c|}{ Parasitoses intestinais* } & \multirow{2}{*}{\multicolumn{2}{|c|}{ Total }} \\
\hline & \multicolumn{2}{|c|}{ Negativo } & \multicolumn{2}{|c|}{ Positivo } & & \\
\hline Adequados $(\geq 20 \mu \mathrm{g} / \mathrm{dL})$ & 40 & 23,4 & 131 & 76,6 & 171 & 100,0 \\
\hline Inadequados $(<20 \mu \mathrm{g} / \mathrm{dL})$ & 11 & 15,7 & 59 & 84,3 & 70 & 100,0 \\
\hline \multirow[t]{2}{*}{ Total } & 51 & 21,2 & 190 & 78,8 & 241 & 100,0 \\
\hline & & 1,32 & $p=0,250$ & & & \\
\hline
\end{tabular}

Obs: *Schistosoma mansoni, Ancylostoma e Ascaris lumbricoides. 
positivos para alguma parasitose. Daqueles portadores de níveis inadequados de retinol, $84,3 \%$ tiveram positividade no exame parasitológico de fezes. Quando analisados para a presença de Schistosoma mansoni, Ancylostoma e Ascaris lumbricoides, não se observou associação significante entre níveis séricos de retinol e parasitoses intestinais $(p=0,250)$.

\section{DIS C U S S Ã O}

A hipovitaminose A tem sido caracterizada como um problema de saúde pública nos países subdesenvolvidos ${ }^{3}$. No Brasil, vários levantamentos realizados em diferentes regiões apontam a hipovitaminose A como um problema endêmico, sobretudo da região Nordeste e Sudeste do país, sendo bem caracterizada em pré-escolares 1,2,18-21.

Os resultados deste estudo indicam que a hipovitaminose A constitui um importante problema de saúde entre os escolares investigados e corroboram aqueles de Araújo et al. ${ }^{1}$, que destacam uma prevalência de 23,6\% de hipovitaminose $\mathrm{A}$ em escolares da área rural de Turmalina, cidade também localizada no Vale do Jequitinhonha.

A constatação da ocorrência de hipovitaminose A no município de Novo Cruzeiro indica a necessidade de intervenção, a exemplo do programa de suplementação de vitamina A que já vem sendo desenvolvido no Nordeste e em alguns municípios do Vale do Jequitinhonha, em Minas Gerais, regiões reconhecidas como "bolsões endêmicos" de deficiência de Vitamina A. Esse programa desenvolvido pelo Ministério da Saúde visa atender às crianças residentes em áreas de risco, associando à suplementação de vitamina $A$ ações educativas implementadas pelos serviços de saúde, disponibilizando informações à população no sentido de selecionar alimentos ricos em retinol e carotenóides para comporem a alimentação diária. No entanto, tais intervenções abrangem somente crianças de 6 a 59 meses, deixando os escolares, como os identificados neste estudo, fora do alvo do programa, não garantindo, assim, a proteção necessária ao longo da vida contra a deficiência de vitamina $A$ e suas conseqüências.

Sendo a vitamina A essencial no combate às infecções, para o crescimento e para prevenção e controle da anemia ${ }^{22}$, a manutenção dos níveis adequados desse micronutriente em diversos estágios da vida, e não somente em pré-escolares, torna-se uma necessidade imperiosa.

Além da carência de vitamina $A$, identificou-se também entre os escolares deste estudo uma alta ocorrência de déficit no crescimento linear e ponderal, embora tal condição não tenha se mostrado associada à hipovitaminose $A$. Resultados similares são registrados por Assis et al. ${ }^{2}$ e Castejon et al. ${ }^{23}$, embora trabalhos como o de Khandait et al. ${ }^{24}$ descrevam a deficiência de vitamina $\mathrm{A}$ associada à desnutrição.

A vulnerabilidade à desnutrição e à deficiência de micronutrientes, incluindo a vitamina $A$, pode ser resultado de uma deficiência alimentar ostensiva. É sabido que para manutenção das reservas hepáticas de vitamina A é necessário um consumo alimentar adequado de suas fontes ${ }^{25}$. Assim, seria esperado que um baixo consumo de vitamina A resultasse na identificação de baixos níveis séricos desse micronutriente. Entre os escolares, verificou-se o baixo consumo de vitamina A e a alta prevalência de hipovitaminose A, mas nenhuma associação entre níveis séricos de retinol e ingestão alimentar de vitamina $A$ foi identificada. Embora essa associação não tenha sido encontrada, a informação sobre o consumo dietético constitui, possivelmente, o único mecanismo de identificação precoce de populações em risco de desenvolver a deficiência de vitamina $A$.

Segundo Batista Filho ${ }^{26}$, o consumo alimentar constitui o elo mais vulnerável da cadeia alimentar e nutricional, e o desemprego, subemprego, oscilações de preço e a baixa renda familiar, dentre outros itens, são fatores que situam milhares de brasileiros abaixo da linha de pobreza e indigência, colocando-os, nesse caso, susceptíveis às mais diversas manifestações por deficiências alimentares, incluindo a hipovitaminose $A$. 
As pessoas que não atingem a renda necessária para adquirir a cesta de alimentos e os bens não alimentares básicos (vestuário, moradia e transporte) são consideradas pobres, pois estão abaixo da linha de pobreza.

Hoffmann ${ }^{27}$ se refere à linha de pobreza adotando dois pontos de corte. Assim, as linhas por ele definidas são a proporção de pessoas com rendimento familiar per capita maior que meio ou maior que um quarto do salário mínimo (ou seja, $R \$ 92,29$ e $R \$ 46,15$, em setembro de 1999, respectivamente).

Nesse sentido, adotando a metodologia de Hoffmann ${ }^{27}$, constatou-se que $97,5 \%$ dos escolares estudados eram, de fato, pobres. Desses, 87,1\% possuíam uma renda menor ou igual a um quarto do salário mínimo. Tais constatações, provavelmente, corroborem os dados do IPEA ${ }^{10}$, que revelaram a existência de 2900 famílias abaixo do nível de pobreza em Novo Cruzeiro no ano de 1993, contribuindo para a lamentável classificação do município no IDH e enfatizando a alta vulnerabilidade dessa população às deficiências nutricionais.

A despeito da relação existente entre pobreza, baixo consumo alimentar e deficiências nutricionais, neste estudo a renda familiar per capita não se mostrou associada aos níveis séricos de retinol. Essa aparente dissociação da carência de vitamina A com uma importante variável socioeconômica merece ser mais investigada.

Além da identificação da hipovitaminose A, défict ponderal e estatural, baixo consumo de fontes de vitamina A e pobreza a que está submetida a população, constatou-se também freqüência elevada de parasitoses entre os escolares quando analisados para a presença de Schistosoma mansoni, Ancylostoma e Ascaris lumbricoides. Embora não tenha sido observada associação estatisticamente significante entre parasitoses e deficientes níveis de vitamina $A$, as evidências indicam que as parasitoses intestinais constituem também problema de saúde entre os escolares estudados, o mesmo podendo ser observado no estudo de Scolari2 ${ }^{28}$, realizado em escolares no município de Ortigueira, no estado do Paraná. Estudos como de Curtale et al. ${ }^{29}$ mostraram a prejudicada absorção de vitamina $A$ em indivíduos portadores de infecção por Ascaris lumbricoides. Friis et al. ${ }^{30}$ verificaram associação entre Schistosoma mansoni e baixas concentrações séricas de retinol. Esses trabalhos enfatizam a importância de considerar que o estado nutricional em relação à vitamina A pode estar de alguma forma associado à presença de parasitas intestinais, não devendo ser descartada a possibilidade de os baixos níveis séricos de retinol aumentarem a susceptibilidade dos indivíduos à infestação ${ }^{30}$.

Os resultados deste estudo colocam em evidência a necessidade de ampliar o diagnóstico da hipovitaminose A para escolares de outras regiões do país, em particular para aqueles que estão submetidos a precárias condições de vida. Os resultados deste estudo reforçam também a importância da ampliação de medidas de suplementação em populações de risco, incluindo os escolares.

\section{A GRADECIMENTOS}

À UFOP, à UFMG, à FUNED, à Diretoria Regional de Saúde de Teófilo Otoni, Secretaria de Saúde de Novo Cruzeiro, Fundação Nacional de Saúde, aos escolares de Novo Cruzeiro, aos professores Marcelo Eustáquio da Silva e Ana Lúcia Rissoni dos Santos e a todos os participantes da coleta de dados.

\section{REFERÊ NCIAS}

1. Araújo RL, Araújo MBDG, Siero RO, Machado RDP, Leite BV. Diagnóstico da hipovitaminose A e anemia nutricional. Estudo realizado na população do Vale do Jequitinhonha, Minas Gerais. Rev Bras Med. 1986; 43(8):225-8.

2. Assis AMO, Prado MS, Freitas MCS, Cruz MM. Deficiência de vitamina A e desnutrição energético- protéica em crianças de localidades do semi-árido baiano. Rev Nutr. 1997; 10(1):70-8.

3. World Health Organization. Indicators for assessing vitamin A deficiency and their application in monitoring and evaluating intervention programmes. Geneva: WHO; 1996. Micronutrient Series. 
4. Tee ES. Carotenoids and retinoids in human nutrition. Crit Rev Food Sci Nutr. 1992; 31(1-2): 103-63.

5. Sedgh G, Herrera MG, Nestel P, El Amin A, Fawzi WW. Dietary vitamin A intake and nondietary factors are associated with reversal of stunting in children. J Nutr. 2000; 130 (10):2520-6.

6. Semba RD. The role of vitamin $A$ and related retinoids in immune function. Nutr Rev. 1998; 56(1 Pt 2): 538-48.

7. World Health Organization Working Group. An evaluation of infant growth: the use and interpretation of antropometry in infants. Bull WHO. 1995; 2(7):165-74.

8. MacLaren DS, Frigg M. Sight and life manual on vitamin A deficiency disorders (VADD). 2nd ed. 2001.

9. Monteiro CA. O mapa da pobreza no Brasil. Dados do IPEA. 1993; 16:18-31.

10. Peliano AMTM, coordenador. O mapa da fome II: informações sobre a indigência por municípios de federação. Brasília: Instituto de Pesquisa Econômica Aplicada; 1993. Documento de Política, nº 15.

11. Instituto Brasileiro de Geografia e Estatística [Internet]. Novo Cruzeiro, Minas Gerais. Disponível em: http:/www.ibge.net/cidadesat/default.php

12. Carr TH, Price EA. Color reactions attributed to vitamin A. Biochem J. 1926; 20:497-501.

13. Interdepartmental Committee on Nutrition for National Defense. Manual for nutrition survey. 2nd ed. Washington (DC): Government Priting Office; 1963.

14. Rosner B, Prineas R, Loggie J, Daniel SR. Percentiles for body mass index in U.S. children 5 to 17 years of age. J Pediatrics. 1998; 132(2):211-22.

15. Margets BM, Cade JE, Osmond C. Comparison of a food frequency questionnaire with a diet record. Int J Epidemiol. 1989; 18(4):868-73.

16. Recommended Dietary Allowances. 10th ed. Washington (DC): National Academy Press; 1989.

17. Pessoa SB, Martins AV. Parasitologia médica. 11 ed. Rio de Janeiro: Guanabara Koogan; 1982.

18. Santos LMP, Dricot JM, Asciuth LS, Dricot-d'ans C. Xerophthalmia in the state of Paraíba, northest of Brazil: clinical findings. Am J Clin Nutr. 1983; 38:139-44.

19. Roncada MJ, Wilson D, Okani ET, Amini S. Prevalência de hipovitaminose $A$ em pré-escolares de município de área metropolitana de São Paulo Brasil. Rev Saúde Pública. 1984; 18:218-24.
20. Araújo RL, Araújo MBDG, Machado RDP, Braga AA, Leite BV, Oliveira JR. Evaluation of a program to overcome vitamin $A$ and iron deficiencies in areas of poverty in Minas Gerais, Brazil. Arch Latinoam Nutr. 1987; 37(1):9-22.

21. Carvalho CMG, Farfan BCW, Venconsky R. Prevalência de hipovitaminose $A$ em crianças da periferia do Município de São Paulo, Brazil. Cad Saúde Pública. 1995; 11(1): 85-96.

22. Mwanri L, Worsley A, Ryan P, Masika J. Supplemental vitamin A improves anemia and growth in anemic school children in Tanzania. J Nutr. 2000; 130(11):2691-6.

23. Castejon HV, Ortega P, Diaz ME, Amaya D, Gomez $G$, Ramos M, et al. Prevalence of sub-clinical vitamin A deficiency and malnutrition in slum children in Maraicabo-Venezuela. Arch Latinoam Nutr. 2001; 51(1):25-32.

24. Khandait DW, Vasudeo ND, Zodpey SP, Kumbhalkar DT, Koram MR. Subclinical vitamin A deficiency in undersix children in Nagpur, India. Southeast Asian J Trop Med Public Health. 1998; 29:289-92.

25. De Pee S, West CE, Permaesih D, Martuti S, Muhila I, Hautvast JGAJ. Orange fruit is more effective than are dark-green, leafy vegetables in increasing serum concentrations of retinol and $\beta$ carotene in schoolchildren in Indonesia. Am J Clin Nutr. 1998; 68(5):1058-67.

26. Batista Filho, M. Projeto fome zero: a importância da divulgação científica de seus resultados. Rev Bras Saúde Materno Infantil. 2003; 3(1):7-8

27. Hoffmann RA. Distribuição de renda no Brasil no período 1993-99 [mimeografado]. Campinas: Universidade Estadual de Campinas, Instituto de Economia; 2001.

28. Scorali C. Prevalence and distribution of soil-transmitted helminth (STH) infections in urban and indigenous schoolchildren in Ortigueira State of Parana, Brasil: implications for control. Trop Med Intern Health. 2000; 4(5):302-7.

29. Curtale EF, Vaidya Y, Muhila I, Tilden RLI. Ascariasis, hookworm infection and serum retinol amongst children in Nepal. Panminerva Med. 1994; 36(1):19-21.

30. Friis $H$, Mwananiki D, Omondi B, Muniv E, Magnussen $P$, Geissler $W$ et al. Serum retinol concentrations and schistosoma mansoni, intestinal helminths and malarial parasitemia: a cross-sectinal study in Kenya preschool and primary school children. Am J Clin Nutr. 1997; 66(3):665-71.

Recebido para publicação em 18 de novembro de 2002 e aceito em 17 de agosto de 2004. 